\title{
Asperuloside stimulates metabolic function in rats across several organs under high-fat diet conditions, acting like the major ingredient of Eucommia leaves with anti-obesity activity
}

\author{
Takahiko Fujikawa ${ }^{1,2}$, Tetsuya Hirata ${ }^{3}$, Shingo Hosoo ${ }^{3}$, Kenji Nakajima ${ }^{3}$, Atsunori Wada ${ }^{3}$, \\ Yutaka Yurugi ${ }^{4}$, Hideaki Soya ${ }^{5}$, Takashi Matsui ${ }^{5}$, Akihiko Yamaguchi ${ }^{6}$, Masato Ogata ${ }^{2}$ and Sansei Nishibe \\ ${ }^{1}$ Laboratory of Molecular Prophylaxis and Pharmacology, Department of Pharmaceutical Sciences, Suruka University of Medical Science, 3500-3 \\ Minamitamagaki-cho, Suzuka, Mie 513-8670, Japan \\ ${ }^{2}$ Department of Biochemistry and Proteomics, Mie University Graduate School of Medicine, 2-174 Edobashi, Tsu, Mie 514-8507, Japan \\ ${ }^{3}$ ReoD Center, Kobayashi Pharmaceutical Co. Ltd, 1-30-3 Toyokawa, Ibaraki, Osaka 567-0057, Japan \\ ${ }^{4}$ Field Science Center of Kii Kuroshio Life Area, Mie University Graduate School of Bioresources, 1577 Kurimamachiya, Tsu, Mie 514-8507, Japan \\ ${ }^{5}$ Laboratory of Exercise Biochemistry, Graduate School of Comprehensive Human Sciences, Tsukuba University, Tsukuba, 1-1-1 Tennoudai, \\ Tsukuba, Ibaraki 305-8574, Japan \\ ${ }^{6}$ Department of Dentistry, Health Sciences University of Hokkaido, 1757 Kanazawa, Tobetsu-cho, Ishikari, Hokkaido 061-0293, Japan \\ ${ }^{7}$ Department of Pharmaceutical Sciences, Health Sciences University of Hokekaido, 1757 Kanazawa, Tobetsu-cho, Ishikari, Hokkaido 061-0293, \\ Japan
}

(Received 17 October 2011 - Final revision received 3 July 2012 - Accepted 6 July 2012)

Journal of Nutritional Science (2012), vol. 1, e10, page 1 of 11

doi:10.1017/jns.2012.12

\section{Abstract}

Eucommia leaves (Eucommia ulmoides Oliver) contain chlorogenic acid (a caffeic acid derivative) and geniposidic acid and asperuloside (ASP), iridoid glucosides used in beverages. We used a metabolic syndrome rat model, produced by feeding a $35 \%$ high-fat diet (HFD), to examine potential anti-obesity and anti-metabolic syndrome effects and mechanisms of chronic administration of ASP. These effects were compared with Eucommia leaf extract (ELE), the positive control, which exhibits anti-obesity effects. A total of six rats were studied for 3 months in five groups. ASP suppressed body weight, visceral fat weight, food intake and circulating levels of glucose, insulin and lipids, and increased the plasma adiponectin level in rats on a HFD. These effects are similar to those of ELE, except for the influence on the plasma glucose level. RT-PCR studies showed that ASP (like ELE with known anti-obesity effects) diminished isocitrate dehydrogenase $3 \alpha$, NADH dehydrogenase flavoprotein 1 (Comp I) mRNA and fatty acid synthase levels (white adipose tissue), increased carnitine palmitoyltransferase $1 \alpha$ and acyl-CoA dehydrogenase, very-long-chain mRNA levels (liver), and increased Glut4, citrate synthase, isocitrate dehydrogenase $3 \alpha$, succinyl CoA synthase, peroxisomal 3-ketoacyl-CoA thiolase, dihydrolipoamide succinyl transferase and succinate dehydrogenase mRNA levels (skeletal muscle) under HFD conditions. Interestingly, ASP administration resulted in significantly increased mRNA levels of uncoupling protein 1 (UCP1) in the brown adipose tissue of HFD-fed rats; ELE did not affect the expression of UCP1. The increased expression of UCP1 may be negated by many ingredients other than ASP in the ELE. These findings suggest that chronic administration of ASP stimulates anti-obesity and anti-metabolic syndrome activity in HFD-fed rats across several organs, similar to ELE administration; thus, ASP may be an important ingredient of ELE.

Key words: Asperuloside: Eucommia ulmoides Oliver: Anti-obesity effects: Metabolic function

\footnotetext{
Abbreviations: Acadvl, acyl-CoA dehydrogenase, very long chain; ASP, asperuloside; BAT, brown adipose tissue; CHA, chlorogenic acid; Comp I, NADH dehydrogenase flavoprotein 1; Comp IV, cytochrome $c$ oxidase, subunit 4a; Cpt1 $\alpha$, carnitine palmitoyltransferase 1 $\alpha$; Cs, citrate synthase; ELE, Eucommia leaf extract; FA, fatty acid; Fas, fatty acid synthase; GEA, geniposidic acid; HFD, high-fat diet; Idh $3 \alpha$, isocitrate dehydrogenase $3 \alpha$; Ogdh, dihydrolipoamide succinyl transferase; Sol. M., soleus muscle; UCP, uncoupling protein; WAT, white adipose tissue.
}

* Corresponding author: Dr T. Fujikawa, fax +81-59-368-1271, email fujikawa@suzuka-u.ac.jp 
Obesity, which can lead to the metabolic syndrome, has recently gained attention as a factor that dramatically increases the risk of arteriosclerotic disease. According to Japanese diagnostic criteria, obesity and the metabolic syndrome are conditions requiring prevention or treatment because of the high risk of complications, including hyperglycaemia, hyperlipaemia and hypertension, due to the accumulation of visceral fat. It has also been reported that accumulated visceral fat tissues secrete various types of adipocytokines and cause metabolic abnormalities such as insulin resistance ${ }^{(1)}$. Because the metabolic syndrome is often triggered by a high-fat diet (HFD) or lack of exercise, the basic treatment of the metabolic syndrome involves lifestyle improvements. There are many dietary and, to a lesser extent, pharmacological approaches that affect energy balance, resulting in successful body weight loss ${ }^{(2)}$. Such therapies typically affect the appetite, nutrient absorption, or thermogenesis related to metabolism, and a recent study indicated that chronic administration of Eucommia leaf extract (ELE) stimulates the metabolic function of rats across several organs under HFD conditions and contributes to anti-obesity or anti-metabolic effects with an improvement of insulin resistance or hyperlipaemia ${ }^{(3)}$.

Eucommia bark, Eucommia ulmoides Oliver (Eucommiaceae), is a herbal medicine traditionally used as an analeptic, analgesic, sedative, antihypertensive and diuretic ${ }^{(4)}$. The commercial product, termed Tochu-cha in Japanese, is composed of roasted Eucommia leaves and has been used in foods. Tochu-cha contains the following active ingredients ${ }^{(5,6)}$ : the iridoid glucosides geniposidic acid (GEA) and asperuloside (ASP) and the caffeic acid derivative chlorogenic acid (CHA). Since the 1970s, Eucommia leaves have been used in the Sichuan District of China as an antihypertensive drug and health $\operatorname{food}^{(7,8)}$, and clinical studies have been conducted to evaluate their efficacy $^{(9-11)}$. Administration of ELE and its ingredients yielded dose-dependent anti-hypertensive effects in spontaneously hypertensive rats ${ }^{(12,13)}$. ELE has also been reported to have potent antioxidant and anti-mutagenic effects ${ }^{(14)}$ and prevents oxidative gastric injury ${ }^{(15)}$. In addition, ELE and its ingredients may inhibit stress-induced gastric erosion ${ }^{(16)}$.

Some previous studies indicated that the anti-obesity effects of ELE are similar to those of powdered leaves ${ }^{(13,17)}$. RT-PCR analysis showed that both roasted and powdered Eucommia leaves enhance metabolic function across several organs, diminishing ATP production (white adipose tissue (WAT)), accelerating $\beta$-oxidation (liver) and increasing the use of ketone bodies/glucose (skeletal muscle). The enhanced metabolic function is accompanied by loss of body weight and visceral fat in rats fed a $\mathrm{HFD}^{(3)}$. Conversely, some studies reported that high-dose administration of Eucommia leaves for $35 \mathrm{~d}^{(18)}$ or low-dose administration for 10 weeks $^{(19)}$ did not significantly decrease the body weight, regardless of the loss and regain of weight in the visceral fat pad in animals. This suggests that differences in the composition of components in Eucommia leaves might have a significant impact on the antiobesity effects observed with ELE. When the anti-obesity effects of compounds in ELE were compared - 0.63\% GEA, $0.45 \%$ ASP and $0.44 \% \mathrm{CHA}$, the relative weights typically found in Eucommia leaves - only ASP exhibited significantly decreased body weight, with decreased visceral fat pad weights as well as decreased plasma levels of NEFA in mice fed a HFD for 4 weeks ${ }^{(20)}$. Administration of $0 \cdot 1 \%$ ASP for 1 month significantly enhanced BMR and resulted in a decreased respiratory quotient that might stimulate lipid metabolism in rats fed a HFD.

The aim of the present study was to examine potential antiobesity effects and the mechanisms of these effects due to chronic administration of ASP, an ingredient of ELE. The effects of the administration of ASP were compared with the effects of the administration of ELE on HFD-fed rats. Furthermore, RT-PCR analysis was used to examine the gene expression across several organs during ASP and ELE administration, including perirenal WAT, liver, soleus muscle (Sol. M.) and brown adipose tissue (BAT) in HFD-fed rats.

\section{Materials and methods}

\section{Animals and diets}

Male Sprague-Dawley rats (4 weeks old; 75-80 g), purchased from SLC, Inc., were maintained at a temperature of $23-26^{\circ} \mathrm{C}$ and a relative humidity of $50-65 \%$ for 2 weeks after arrival. Rats were divided into five groups (HFD-Control, HFD-5 $\%$ ELE, HFD-0.03\% ASP, HFD-0.1\% ASP and HFD-0.3\% ASP; $n 6$ for each) based on body weight. Under HFD (2.6 MJ/100 g) conditions, all animals were fed a commercial diet containing 29.7\% lard (Oriental Yeast Co. Ltd.), water $(7.7 \%)$, protein $(23.6 \%)$, lipid $(35.0 \%)$, ash $(6.1 \%)$, dietary fibre $(2.9 \%)$ and nitrogen-free extract $(24.7 \%)$. Test foods were prepared by adding $5 \%$ ELE and $0 \cdot 03-0.3 \%$ ASP or by adding $5 \%$ casein as a control to determine the effects of high-dose administration in rats. The test foods were provided ad libitum for 3 months to examine the potential anti-obesity and anti-metabolic syndrome effects of ELE and ASP. The doses given to animals fed a HFD were determined based on the findings of Fujikawa et al. ${ }^{(3)}$. After chronic administration of ELE and ASP, body weight was measured, and the rats were killed by decapitation without stress. WAT (perirenal WAT and epididymal WAT), BAT and skeletal muscle (the Sol. M. of the hind leg) were immediately removed.

The Institutional Animal Care and Use Committees at Mie University Faculty of Medicine and the Suzuka University of Medical Science approved the animal facilities and study protocols. All procedures were performed in accordance with the National Institutes of Health guidelines for animal care (1996).

\section{Preparation of Eucommia leaf extract}

Eucommia leaves (Eucommia ulmoides Oliver) collected in the Sichuan District of China were used. ELE was prepared as follows: fresh Eucommia leaves were treated with steam at 100$110^{\circ} \mathrm{C}$ and then dried and roasted. A quantity of 2 tonnes of roasted Eucommia leaves was steeped in 10 tonnes of hot water at $90^{\circ} \mathrm{C}$ for $1 \mathrm{~h}$, and the extract was filtered and concentrated. The concentrate was left standing for $1 \mathrm{~d}$. The concentrate was then filtered and concentrated, vacuum-dried and powdered (yield: $18 \%$ ). 
We measured each of the major components (i.e. GEA, ASP and CHA) in each sample using HPLC with an ODS col$\mathrm{umn}^{(20)}$. The experimentally used ELE was composed of GEA $(63.0 \mathrm{mg} / \mathrm{g})$, ASP $(45.2 \mathrm{mg} / \mathrm{g})$ and CHA $(44.0 \mathrm{mg} / \mathrm{g})$.

\section{Preparation of asperuloside}

ASP was extracted and isolated from Eucommia leaves. Dried leaves of Eucommia were extracted with hot water for $10 \mathrm{~h}$ at $60^{\circ} \mathrm{C}$, vacuum-dried and powdered to obtain the ELE. The extract was subjected to Diaion HP-20P column chromatography with a gradient of water-methanol (1:0 to $0: 1)$ to yield water fractions, $30 \%$ methanol fractions, $50 \%$ methanol fractions, $80 \%$ methanol fractions and $100 \%$ methanol fractions in the order of elution. The $30 \%$ methanol fractions were chromatographed over YMC S-15/30 120A ODS with a gradient of water-methanol (1:1 to 6:1) and then purified by Daisogel SP-120-40/60-ODS-B $(100 \times 1000 \mathrm{~mm})$, eluted with $80 \%$ methanol, vacuum-dried and freeze-dried, and washed with acetone to yield ASP (purity: 99.5\%).

\section{Body weight and percentage white adipose tissue weight and skeletal muscle weight relative to the body weight}

Body weight was measured weekly throughout the experimental period. After killing, the perirenal WAT, epididymal WAT, BAT and Sol. M. of the hind leg were removed, and the wet weights were measured. Weights were calculated as percentage of wet weight to body weight.

\section{Blood components}

Blood was collected immediately after the animals had been killed by decapitation. The blood was centrifuged $(3000 \mathrm{rpm}$, $30 \mathrm{~min}$ ), and the separated plasma was stored at $-80^{\circ} \mathrm{C}$ until measurement. Plasma glucose and insulin were measured as biomarkers of carbohydrate metabolism; total cholesterol, TGA and NEFA in the plasma were measured as biomarkers of lipid metabolism. Plasma levels of adipocytokines (adiponectin and TNF- $\alpha$ ) were also measured. Insulin levels were measured by ELISA using an ultra-high-sensitivity rat insulin assay kit (Morinaga Milk Industry Co. Ltd.), adiponectin levels were measured using a mouse/rat adiponectin ELISA kit (Otsuka Pharmaceutical Co. Ltd), and TNF- $\alpha$ levels were measured using a rat TNF- $\alpha$ assay kit (Immuno-Biological Laboratories Co. Ltd.). Other parameters were measured using a Hitachi 7180 automated analyser.

\section{Hepatic lipid metabolic enzyme activity}

Frozen rat liver was thawed and homogenised on ice with six volumes of a $0.25 \mathrm{M}$-sucrose solution containing $1 \mathrm{~mm}$-EDTA and $3 \mathrm{~mm}-\mathrm{Tris}-\mathrm{HCl}(\mathrm{pH} 7.2)$ and centrifuged at $500 \mathrm{~g}$ for 10 $\min \left(4^{\circ} \mathrm{C}\right)$. The supernatant was centrifuged at $9000 \mathrm{~g}$ for 10 min, followed by centrifugation of the resultant supernatant at $105000 \mathrm{~g}$ for $1 \mathrm{~h}$. The supernatant containing the cytosol was used for the measurement of fatty acid synthase (Fas) activity. After spinning at $500 \mathrm{~g}$, the pellet containing the mitochondria was suspended in a small volume of a $0.25 \mathrm{M}$-sucrose solution. After centrifugation at $9000 \mathrm{~g}$ for $10 \mathrm{~min}$, the supernatant was used to measure carnitine palmitoyltransferase $1 \alpha$ (Cpt1 $\alpha)$ activity. Fas activity was determined using a method with extensive modification based on that described by Kelley et al. ${ }^{(21)}$. The cytosolic fraction $(20-100 \mu \mathrm{l})$ was mixed with $500 \mu \mathrm{l}$ of $0.2 \mathrm{M}$-potassium phosphate buffer ( $\mathrm{pH} 7 \cdot 0$ ), $20 \mu \mathrm{l}$ of 2.5 mM-acetyl-CoA, $30 \mu \mathrm{l}$ of $10 \mathrm{~mm}-\mathrm{NADPH}$ and $20 \mu \mathrm{l}$ of 10 mM-malonyl-CoA and then measured at $340 \mathrm{~nm}\left(30^{\circ} \mathrm{C}\right)$ for 2 $3 \mathrm{~min}$ in a spectrophotometer. Cpt1 $\alpha$ activity was determined by a method with extensive modification based on that described by Markwell et al. ${ }^{(22)}$. The mitochondrial fraction $(5-15 \mu \mathrm{l})$ was mixed with $500 \mu \mathrm{l}$ of $116 \mathrm{~mm}-\mathrm{Tris}-\mathrm{HCl}(\mathrm{pH} 8 \cdot 0)$ buffer, 2.5 mм-EDTA, $5 \mu \mathrm{m}-5,5$-dithiobis-2-nitrobenzoate (DTNB), $0 \cdot 2 \%$ Triton X-100, 2 mm-palmitoyl-CoA and 1.25 mm-L-carnitine. The reaction was initiated by the addition of enzyme at $25^{\circ} \mathrm{C}$, and the rate of $\mathrm{CoA}$ release from palmitoyl-CoA was measured at $412 \mathrm{~nm}$ using spectrophotometry.

\section{$R T-P C R$}

Total RNA was extracted using a TriPure Isolation kit (Roche), and the RNA concentrations were determined spectrophotometrically at $260 \mathrm{~nm}$. The quality of the RNA was determined spectrophotometrically using a $260 / 280 \mathrm{~nm}$ absorbance ratio; the values of all the samples were 1.8-2.0. After total RNA extraction, the samples were treated with a TURBO DNA-free kit (Ambion) for $30 \mathrm{~min}$ at $37^{\circ} \mathrm{C}$ to remove any genomic DNA. The DNase-treated RNA $(0.35 \mu \mathrm{g})$ was utilised to synthesise first-strand cDNA using the ReverTraAce qPCR RT kit (Toyobo). The cDNA products $(50 \mathrm{ng} / \mu \mathrm{l})$ were analysed by RT-PCR using Power SYBR Green PCR Master Mix (Applied Biosystems) with an ABI PRISM 7300 (Applied Biosystems). The amplification program included an initial denaturation step at $95^{\circ} \mathrm{C}$ for $10 \mathrm{~min}$, forty cycles of denaturation at $95^{\circ} \mathrm{C}$ for $15 \mathrm{~s}$ and annealing/extension at $59-60^{\circ} \mathrm{C}$ for $1 \mathrm{~min}$. The glyceraldehyde-3-phosphate dehydrogenase mRNA level was determined and used as an internal control. Each gene was normalised to glyceraldehyde-3-phosphate dehydrogenase by subtracting the cycle threshold $(\mathrm{Ct})$ value of glyceraldehyde-3phosphate dehydrogenase from the $\mathrm{Ct}$ value of the gene target $(\Delta \mathrm{Ct}[$ target $])$. The relative expression of the target gene was calculated using SDS v1.2 with the relative quantification (respiratory quotient) software (Applied Biosystems), with $\Delta \mathrm{Ct}$ [target] compared with the $\Delta \mathrm{Ct}$ values of the reference; i.e., $\Delta \Delta \mathrm{Ct}$ $=\Delta \mathrm{Ct}[$ target $]-\Delta \mathrm{Ct}[$ reference $]$. The degree of difference (expressed in fold difference) between the target and the reference was calculated as $2^{-\Delta \Delta C t}$. The melting point analysis detected no non-specific amplification in the cDNA samples. The slopes of the amplification curves did not differ between groups during the mRNA analysis, and no differences in the amplification efficiency were observed. The sequences of the specific primers used in the Power SYBR Green PCR Master Mix protocol are listed in Supplementary Table S1 (available online at http://journals.cambridge.org/JNS). Each PCR primer was designed using DINASIS Pro v2.7 software (Hitachi Software Engineering Co. Ltd), and the oligonucleotides were purchased from Greiner Bio-One Co. Ltd. 


\section{Statistical analysis}

The values are expressed as means with their standard errors derived from measurements of six rats $(n 6)$. Statistical analysis was performed using SPSS statistics 20 (IBM). The equal variances were checked by Levene's test. One-way ANOVA was used for inter-group comparisons and for linear trend and quadratic trend tests. When the ANOVA results revealed significant differences, contrast tests were used to identify significant differences from HFD-5 \% ELE or HFD-Control group. In the statistics for each contrast test, we preplanned to leave out the cubic response but compared $5 \%$ ELE or Control to the average of the three ASP treatments. This is assuming that we do not have a specific ASP treatment that we expect to compare to $5 \%$ ELE a priori: coefficients for linear $(-0.4597, \quad-0.3314,-0.0321 \quad 0.8233)$ and quadratic $(0.5334,0.00171,-0.8025,0.2673)$ responses with Control $(0 \%), 0.03 \%$ ASP, $0.1 \%$ ASP and $0.3 \%$ ASP; coefficients for Control v. $5 \%$ ELE $(1,0,0,0,-1)$ and ASP $v .5 \%$ ELE $(0-1,-1,-1,3)$ with Control, $0.03 \%$ ASP, $0.1 \%$ ASP, $0.3 \%$ ASP and $5 \%$ ELE.

\section{Results}

\section{Body weight, food intake and relative weights of white adipose tissue, brown adipose tissue and soleus muscle with chronic administration of Eucommia leaf extract and asperuloside}

Compared with the HFD-Control group, final body weight, body weight gain and food intake decreased significantly in 3 months in the HFD-5\% ELE group (each $P<0.05$ ), and the dose-dependent decrease was observed in the HFD- $0.03-0.3 \%$ ASP group compared with the
HFD-Control group (each $P<0 \cdot 05$, Table 1). No difference in the final body weight, body weight gain and food intake was observed between the HFD-5\% ELE and the average of all HFD-0.1-0.3\% ASP groups (Table 1).

Compared with the HFD-Control group, the relative weight of the perirenal WAT significantly decreased in 3 months in the HFD-5\% ELE group $(P<0.05)$, and a significant and dose dependent decrease was observed in the HFD-0.03$0.3 \%$ ASP group when compared with the HFD-Control group $(P<0 \cdot 05$, Table 1$)$. These changes in the ASP groups were dose-dependent $(P<0 \cdot 05$, Table 1$)$. No difference in the relative weight of the perirenal WAT was observed between the HFD-5\% ELE and the average of the HFD- $0 \cdot 1-0 \cdot 3 \%$ ASP groups (Table 1). The relative BAT weight increased in the HFD-5 \% ELE group under chronic administration $(P<0.05$, Table 1). The ASP groups exhibited a significant increase with linearity when compared with the HFD-Control group $(P<0 \cdot 05$, Table 1$)$. A significant difference in relative BAT weight was observed between the HFD-5\% ELE and the average of the three HFD-ASP groups $(P<0 \cdot 05$, Table 1). The relative Sol. M. weights were not significantly different between groups (Table 1).

The control group exhibited primary signs of progressive metabolic syndrome with plasma parameters in agreement with a previous report ${ }^{(3)}$.

\section{Plasma levels of lipids, glucose and insulin with chronic administration of Eucommia leaf extract and asperuloside}

In comparison with the HFD-Control group, plasma NEFA levels significantly decreased in the HFD-5\% ELE group during 3 months of HFD feeding $(P<0 \cdot 05$, Table 1$)$. The

Table 1. Effects of asperuloside (ASP) on body weight, white adipose tissue (WAT) weight, brown adipose tissue (BAT) weight, soleus muscle (Sol. M) weight and plasma indices in high-fat diet (HFD)-fed rats

(Mean values with their standard errors)

\begin{tabular}{|c|c|c|c|c|c|c|c|c|c|c|}
\hline & \multirow{2}{*}{\multicolumn{2}{|c|}{ HFD-Cont. $(n 6)$}} & \multicolumn{6}{|c|}{ HFD-ASP } & \multirow{2}{*}{\multicolumn{2}{|c|}{$\begin{array}{l}\text { HFD-ELE } \\
5 \%(n 6)\end{array}$}} \\
\hline & & & \multicolumn{2}{|c|}{$0.03 \%(n 6)$} & \multicolumn{2}{|c|}{$0.1 \%(n 6)$} & \multicolumn{2}{|c|}{$0.3 \%(n 6)$} & & \\
\hline & Mean & $\mathrm{SE}$ & Mean & SE & Mean & SE & Mean & SE & Mean & $\mathrm{SE}$ \\
\hline Initial body weight (g per rat) & $71 \cdot 0$ & 1.0 & $71 \cdot 2$ & 1.5 & $72 \cdot 5$ & 0.5 & $71 \cdot 0$ & 0.6 & 71.7 & 0.7 \\
\hline Food intake (g/d per rat) ${ }^{*} \dagger$ & $27 \cdot 8$ & $2 \cdot 2$ & $21 \cdot 3$ & $3 \cdot 2$ & $17 \cdot 7$ & $2 \cdot 7$ & 14.9 & $2 \cdot 0$ & $15 \cdot 6$ & 1.6 \\
\hline Final body weight (g per rat)* ${ }^{*}$ & 564 & 9 & 516 & 19 & 465 & 8 & 461 & 7 & 462 & 17 \\
\hline Body weight gain (g per rat) ${ }^{*} \dagger$ & 493 & 10 & 445 & 18 & 393 & 8 & 390 & 7 & 390 & 17 \\
\hline \multicolumn{11}{|l|}{ Relative WAT weight (\%) } \\
\hline Perirenal $^{*} \dagger$ & $2 \cdot 7$ & 0.3 & 1.5 & 0.2 & 1.4 & 0.1 & 1.3 & 0.1 & 1.5 & 0.1 \\
\hline Epididymal & $2 \cdot 6$ & 0.2 & 2.5 & 0.2 & $2 \cdot 2$ & 0.1 & $2 \cdot 0$ & 0.1 & $2 \cdot 0$ & 0.2 \\
\hline Relative BAT weight $(\%)^{*}+\neq$ & 0.24 & 0.02 & 0.31 & 0.01 & 0.33 & 0.02 & 0.37 & 0.02 & 0.39 & 0.04 \\
\hline Relative Sol. M. weight (\%) & 0.07 & 0.01 & 0.07 & 0.01 & 0.07 & 0.01 & 0.07 & 0.01 & 0.07 & 0.01 \\
\hline Glucose $\left(\mathrm{mg} / \mathrm{l}^{*} \dagger\right.$ & 1621 & 71 & 1501 & 37 & 1394 & 42 & 1338 & 55 & 1413 & 63 \\
\hline Insulin $(\mathrm{ng} / \mathrm{ml})^{\star}+$ & 7.7 & 0.6 & $5 \cdot 2$ & $1 \cdot 1$ & 3.9 & 0.8 & 3.3 & 0.6 & 3.4 & 0.7 \\
\hline TAG $\left(\mathrm{mg} / \mathrm{l}^{*} \dagger\right.$ & 2260 & 378 & 1605 & 301 & 1199 & 235 & 1071 & 243 & 1143 & 237 \\
\hline $\operatorname{NEFA}(\mu \mathrm{Eq} / \mathrm{l})^{*} \dagger$ & $639 \cdot 1$ & 33.7 & 449.0 & $56 \cdot 0$ & $402 \cdot 7$ & $21 \cdot 6$ & $397 \cdot 3$ & $20 \cdot 9$ & $437 \cdot 2$ & $30 \cdot 3$ \\
\hline Total cholesterol $\left(\mathrm{mg} / \mathrm{l}^{*} \dagger \ddagger\right.$ & 880 & 34 & 721 & 25 & 708 & 24 & 664 & 26 & 591 & 28 \\
\hline Adiponectin $(\mu \mathrm{g} / \mathrm{l})^{\star} \dagger$ & 29 & 5 & 39 & 6 & 48 & 4 & 53 & 3 & 54 & 6 \\
\hline TNF- $\alpha(p g / m l)^{*} \dagger$ & $198 \cdot 3$ & $18 \cdot 2$ & 136.5 & $13 \cdot 1$ & 98.7 & $9 \cdot 2$ & $70 \cdot 6$ & 8.9 & 68.9 & $10 \cdot 1$ \\
\hline
\end{tabular}

ELE, Eucommia leaf extract; Cont., Control.

* Linear contrast with Control $(0 \%), 0.03 \%, 0.1 \%$ and $0.3 \%$ ASP $(P<0.05)$.

† Contrast for Control v. $5 \%$ ELE $(P<0.05)$.

$\neq$ Contrast for the average of all ASP treatments v. $5 \%$ ELE $(P<0.05)$. 
ASP groups exhibited a significant decrease with linearity when compared with the HFD-Control group $(P<0 \cdot 05$, Table 1). Administration of ASP dose dependently reduced the plasma TAG level in rats fed a HFD when compared with the HFD-Control rats $(P<0.05)$, and a significant decrease was seen in the HFD-5 \% ELE group $(P<0.05$, Table 1$)$. No difference in the plasma level of NEFA and TAG was observed between the HFD-5\% ELE and the average of all HFD-0.1-0.3\% ASP groups (Table 1). A significantly decreased total cholesterol level was observed in $5 \%$ ELE-administered rats under HFD conditions $(P<0 \cdot 05$, Table 1). The ASP groups exhibited a significant decrease of the plasma total cholesterol level in a dose-dependent fashion when compared with the HFD-Control group $(P<0.05)$, and a significant difference was observed between the HFD-5\% ELE and the average of the HFD-0.1-0.3\% ASP groups $(P<0 \cdot 05$, Table 1$)$. A dose-dependently reduced plasma glucose level was observed in the HFD-ASP groups compared with the HFD-Control group after 3 months of HFD feeding $(P<0 \cdot 05)$; however, no difference in plasma glucose level was observed between the HFD-5 \% ELE and the average of the three HFD-ASP groups (Table 1). Administration of $5 \%$ ELE yielded markedly decreased plasma insulin levels under HFD conditions $(P<0 \cdot 05)$, and linearly decreased plasma insulin levels were also observed in rats administered $0.1-0.3 \%$ ASP contrasted with the HFD-Control rats $(P<0.05$, Table 1). No difference in the plasma insulin level was observed between the HFD-5\% ELE and the average of the HFD-ASP groups (Table 1).

\section{Plasma adipocytokine levels with chronic administration of} Eucommia leaf extract and asperuloside

In the HFD-5 \% ELE group, significantly increased levels of plasma adiponectin were observed; a dose-dependent increase was also observed in the HFD-ASP groups compared with the HFD-Control group (each $P<0 \cdot 05$, Table 1). No difference in the plasma level of adiponectin was observed between the HFD-5\% ELE and the average of the three HFD-ASP groups (Table 1).

In contrast, administration of $5 \%$ ELE yielded a considerably decreased plasma TNF- $\alpha$ level under HFD conditions; a dose-dependently decreased TNF- $\alpha$ level was also observed in the ASP-administered rats under HFD conditions compared with the HFD-Control rats (each $P<0 \cdot 05$, Table 1). No difference in the plasma level of TNF- $\alpha$ was observed between the HFD- $5 \%$ ELE and the average of the HFD-0.1-0.3\% ASP groups (Table 1).

\section{Gene expression related to glucose metabolism with chronic administration of Eucommia leaf extract and asperuloside}

Under HFD conditions, chronic administration of $5 \%$ ELE significantly induced the expression of glucokinase, citrate synthase (Cs), dihydrolipoamide succinyl transferase (Ogdh), NADH dehydrogenase flavoprotein 1 (Comp I) and cytochrome $c$ oxidase, subunit $4 \mathrm{a}$ (Comp IV) in the rat liver (each $P<0.05$, Table 2). The ASP groups exhibited a significant and dosedependent increase of glucokinase, Cs, Ogdh, Comp I and

Table 2. Gene expression analysis by real-time PCR in liver after administration of asperuloside (ASP) under high-fat diet (HFD) conditions (Mean values with their standard errors)

\begin{tabular}{|c|c|c|c|c|c|c|c|c|c|c|}
\hline \multirow[b]{3}{*}{ Gene name } & \multirow{2}{*}{\multicolumn{2}{|c|}{ HFD-Cont. (n 6) }} & \multicolumn{6}{|c|}{ HFD-ASP (fold of Cont.) } & \multirow{2}{*}{\multicolumn{2}{|c|}{$\begin{array}{c}\begin{array}{c}\text { HFD-ELE } \\
\text { (fold of Cont.) }\end{array} \\
5 \%(n 6)\end{array}$}} \\
\hline & & & \multicolumn{2}{|c|}{$0.03 \%(n 6)$} & \multicolumn{2}{|c|}{$0.1 \%(n 6)$} & \multicolumn{2}{|c|}{$0.3 \%(n 6)$} & & \\
\hline & Mean & SE & Mean & SE & Mean & SE & Mean & SE & Mean & SE \\
\hline \multicolumn{11}{|c|}{ Glycolytic system } \\
\hline Gck $^{*} \dagger$ & 1.00 & 0.11 & 1.47 & 0.11 & 1.82 & 0.15 & $2 \cdot 19$ & 0.25 & $2 \cdot 60$ & 0.42 \\
\hline \multicolumn{11}{|l|}{ TCA cycle } \\
\hline$C s^{*}+t$ & 1.00 & 0.11 & 0.96 & 0.04 & 1.90 & 0.07 & 1.97 & 0.08 & $2 \cdot 15$ & 0.08 \\
\hline Ogdh* $+\ddagger$ & 1.00 & 0.12 & 0.98 & 0.08 & 1.49 & 0.14 & 1.78 & 0.08 & $2 \cdot 89$ & 0.05 \\
\hline \multicolumn{11}{|c|}{ Electron transfer system } \\
\hline Comp I*t‡ & 1.00 & 0.10 & 0.96 & 0.06 & 1.42 & 0.10 & 2.06 & 0.10 & 2.39 & 0.08 \\
\hline Comp IV*t‡ & 1.00 & 0.12 & 1.05 & 0.06 & 0.84 & 0.11 & 0.61 & 0.08 & 0.62 & 0.07 \\
\hline \multicolumn{11}{|c|}{ Fatty acid synthesis } \\
\hline $\mathrm{Fas}^{*}+$ & 1.00 & $0 \cdot 10$ & 1.24 & 0.13 & 1.67 & 0.17 & 1.76 & 0.03 & 2.05 & 0.22 \\
\hline \multicolumn{11}{|c|}{ Fatty acid transporter } \\
\hline Fatp* $†$ & 1.00 & 0.10 & $1 \cdot 24$ & 0.13 & 1.67 & 0.17 & 1.76 & 0.03 & 2.05 & 0.22 \\
\hline \multicolumn{11}{|c|}{ Fatty acid $\beta$-oxidation } \\
\hline Cptl $\alpha^{*} \dagger$ & 1.00 & $0 \cdot 10$ & 1.23 & 0.15 & 1.57 & $0 \cdot 10$ & 1.76 & 0.16 & 2.35 & 0.07 \\
\hline Acadvi* $† \ddagger$ & 1.00 & 0.10 & $1 \cdot 22$ & 0.05 & 1.37 & 0.06 & 2.05 & 0.12 & $1 \cdot 84$ & 0.13 \\
\hline \multicolumn{11}{|c|}{ Fatty acid $\beta$-oxidation (catalytic) } \\
\hline Hadh II*十† & 1.00 & 0.15 & 1.35 & 0.07 & 1.77 & 0.17 & $2 \cdot 32$ & 0.20 & $2 \cdot 27$ & 0.16 \\
\hline Hadh $S^{*}+t$ & 1.00 & 0.12 & 0.95 & $0.07^{\prime}$ & 1.49 & 0.12 & 1.80 & 0.10 & 1.72 & 0.09 \\
\hline
\end{tabular}

Cont., Control; ELE, Eucommia leaf extract; Gck, glucokinase; Cs, citrate synthase; Ogdh, dihydrolipoamide succinyl transferase; Comp I, NADH dehydrogenase flavoprotein 1; Comp IV, cytochrome $c$ oxidase, subunit 4a; Fas, fatty acid synthase; Fatp, fatty acid transport protein; Cpt1 $\alpha$, carnitine palmitoyltransferase 1 $\alpha$; Acadvl, acyl-CoA dehydrogenase, very long chain; Hadh II, hydroxyacyl-CoA dehydrogenase type II; Hadh SC, L-3-hydroxyacyl-CoA dehydrogenase, short chain.

* Linear contrast with Control $(0 \%), 0.03 \%, 0.1 \%$ and $0.3 \%$ ASP $(P<0.05)$.

† Contrast for Control v. $5 \%$ ELE $(P<0.05)$.

$\ddagger$ Contrast for the average of all ASP treatments v. $5 \%$ ELE $(P<0.05)$. 
Comp IV mRNA when compared with the HFD-Control group $(P<0 \cdot 05)$, and the significantly increased mRNA level of $\mathrm{Cs}$, Ogdh, Comp I and Comp IV in the average of all the HFD-0.03-0.3\% ASP groups was lower than that of the HFD-5 \% ELE group $(P<0 \cdot 05$, Table 2$)$. A significant difference in glucokinase mRNA level was not observed between the HFD-5 \% ELE and the average of the HFD-0.03-0.3\% ASP groups (Table 2). In the perirenal WAT of rats fed a HFD, the levels of isocitrate dehydrogenase $3 \alpha(\operatorname{Idh} 3 \alpha)$ and Comp I mRNA were markedly decreased by chronic administration of $5 \%$ ELE (each $P<0 \cdot 05$, Table 3 ). The ASP groups exhibited a significant decrease of Glut4, Idh $3 \alpha$ and Comp I mRNA with linearity when compared with the HFD-Control group (each $P<0.05$, Table 3 ). No difference was observed between the HFD-5\% ELE and the average of the three HFD-ASP groups (Table 3). In the Sol. M. of rats under HFD conditions, the $5 \%$ ELE group had significantly increased levels of Glut4, Cs, Idh $3 \alpha$, Ogdh, succinate dehydrogenase complex, subunit A, flavoprotein, Comp I, Comp IV and ATP synthase, $\mathrm{H}^{+}$transporting, mitochondrial F1 complex, delta subunit (Comp V) mRNA (each $P<0 \cdot 05$, Table 4). Similarly, the ASP groups had a markedly increased mRNA level of these genes with linearity when compared with the HFD-Control group (each
$P<0 \cdot 05$, Table 4). A difference except for Idh3 $\alpha$ mRNA was observed between the HFD-5\% ELE and the average of the HFD-ASP groups (each $P<0 \cdot 05$, Table 4).

\section{Gene expression related to lipid metabolism with chronic administration of Eucommia leaf extract and asperuloside}

In the rat liver under HFD conditions, chronic administration of $5 \%$ ELE significantly decreased the level of expression of Fas mRNA and a marked increased the mRNA levels of FA transport protein, Cpt1 $\alpha$, acyl-CoA dehydrogenase, very long chain (Acadvl), hydroxyacyl-CoA dehydrogenase type II and L-3-hydroxyacyl-CoA dehydrogenase, short chain (each $P<0 \cdot 05$, Table 2$)$. The HFD-ASP groups also exhibited a significant decrease in Fas mRNA and markedly increase in these mRNA in the liver in a dose-dependent fashion when compared with the HFD-Control group (each $P<0 \cdot 05$, Table 2). Significant differences in Acadvl, hydroxyacyl-CoA dehydrogenase type II and L-3-hydroxyacyl-CoA dehydrogenase, short chain mRNA levels were observed between the HFD-5 \% ELE and the average of all the HFD-0.03-0.3\% ASP groups $(P<0 \cdot 05)$, but not in Fas, FA transport protein and $\mathrm{Cpt} 1 \alpha$ mRNA levels (Table 2). The HFD-5\% ELE

Table 3. Gene expression analysis by real-time PCR in perirenal white adipose tissue after administration of asperuloside (ASP) under high-fat diet (HFD) conditions

(Mean values with their standard errors)

\begin{tabular}{|c|c|c|c|c|c|c|c|c|c|c|}
\hline \multirow[b]{3}{*}{ Gene name } & & & \multicolumn{6}{|c|}{ HFD-ASP (fold of Cont.) } & \multirow{2}{*}{\multicolumn{2}{|c|}{$\begin{array}{c}\begin{array}{c}\text { HFD-ELE } \\
\text { (fold of Cont.) }\end{array} \\
5 \%(n 6)\end{array}$}} \\
\hline & \multicolumn{2}{|c|}{ HFD-Cont. (n 6) } & \multicolumn{2}{|c|}{$0.03 \%(n 6)$} & \multicolumn{2}{|c|}{$0.1 \%(n 6)$} & \multicolumn{2}{|c|}{$0.3 \%(n 6)$} & & \\
\hline & Mean & SE & Mean & SE & Mean & SE & Mean & SE & Mean & SE \\
\hline \multicolumn{11}{|l|}{ GLUT } \\
\hline Glut4* & 1.00 & 0.08 & 0.80 & 0.07 & 0.66 & 0.04 & 0.57 & 0.09 & 0.79 & 0.10 \\
\hline \multicolumn{11}{|l|}{ Glycolytic system } \\
\hline Hk2 & 1.00 & 0.07 & 0.95 & 0.18 & 0.78 & 0.06 & 0.66 & 0.04 & 1.06 & 0.20 \\
\hline \multicolumn{11}{|l|}{ TCA cycle } \\
\hline Cs & 1.00 & 0.04 & $1 \cdot 10$ & 0.14 & 0.81 & 0.05 & 0.67 & 0.04 & 0.98 & 0.14 \\
\hline $\operatorname{ldh} 3 \alpha^{*} \dagger$ & 1.00 & 0.06 & 1.02 & 0.14 & 0.89 & 0.09 & 0.57 & 0.09 & 0.60 & 0.07 \\
\hline Ogdh & 1.00 & 0.12 & 1.11 & 0.18 & 0.69 & 0.04 & 0.93 & 0.33 & 0.93 & 0.25 \\
\hline \multicolumn{11}{|c|}{ Electron transfer system } \\
\hline Comp I* & 1.00 & 0.05 & 1.03 & 0.11 & 0.66 & 0.02 & 0.58 & 0.03 & 0.64 & 0.07 \\
\hline Comp IV & 1.00 & 0.08 & $1 \cdot 13$ & 0.09 & 1.00 & 0.06 & 0.64 & $0 \cdot 27$ & 0.99 & 0.26 \\
\hline \multicolumn{11}{|l|}{ Lipolysis } \\
\hline$\left.\mathrm{Hs}\right|^{*}$ & 1.00 & 0.17 & 1.73 & 0.43 & 2.09 & 0.48 & 2.73 & 0.21 & 1.76 & 0.49 \\
\hline \multicolumn{11}{|c|}{ Fatty acid synthesis } \\
\hline Fas $^{*}+t$ & 1.00 & 0.05 & 0.67 & 0.03 & 0.48 & 0.04 & 0.42 & 0.04 & 0.69 & 0.05 \\
\hline \multicolumn{11}{|c|}{ Fatty acid transporter } \\
\hline Fatp & 1.00 & 0.06 & 0.88 & 0.05 & 0.85 & 0.05 & 0.52 & 0.05 & 0.63 & 0.06 \\
\hline \multicolumn{11}{|c|}{ Fatty acid ß-oxidation } \\
\hline Cpt2 & 1.00 & 0.11 & 1.25 & 0.19 & 1.44 & $0 \cdot 13$ & 1.71 & 0.32 & 1.61 & 0.18 \\
\hline Acadvl ${ }^{*} \dagger$ & 1.00 & 0.10 & 1.43 & 0.07 & 1.77 & 0.26 & $2 \cdot 70$ & 0.28 & 1.93 & 0.09 \\
\hline \multicolumn{11}{|c|}{ Fatty acid receptor and Adipocytokine } \\
\hline PPAR $\gamma^{*} \dagger$ & 1.00 & 0.09 & 1.18 & 0.02 & 1.80 & 0.12 & $2 \cdot 11$ & 0.08 & 1.98 & 0.12 \\
\hline Adiponectin* $\dagger$ & 1.00 & 0.11 & 1.01 & 0.02 & $2 \cdot 38$ & 0.22 & 3.03 & 0.14 & 2.47 & 0.11 \\
\hline Resistin* $†$ & 1.00 & 0.05 & 0.97 & 0.07 & 0.86 & 0.04 & 0.66 & 0.05 & 0.68 & 0.08 \\
\hline
\end{tabular}

Cont., Control; ELE, Eucommia leaf extract; Hk2, hexokinase 2; Cs, citrate synthase; Idh3 $\alpha$, isocitrate dehydrogenase $3 \alpha$; Ogdh, dihydrolipoamide succinyl transferase; Comp I, $\mathrm{NADH}$ dehydrogenase flavoprotein 1; Comp IV, cytochrome $c$ oxidase, subunit 4a; Hsl, hormone-sensitive lipase; Fas, fatty acid synthase; Fatp, fatty acid transporter protein;

Cpt2, carnitine palmitoyltransferase 2; Acadvl, acyl-CoA dehydrogenase, very long chain.

* Linear contrast with Control $(0 \%), 0.03 \%, 0.1 \%$ and $0.3 \%$ ASP $(P<0.05)$.

† Contrast for Control v. $5 \%$ ELE $(P<0.05)$.

$\ddagger$ Contrast for the average of all ASP treatments v. $5 \%$ ELE $(P<0.05)$. 
Table 4. Gene expression analysis by real-time PCR in soleus muscle after administration of asperuloside (ASP) under high-fat diet (HFD) conditions (Mean values with their standard errors)

\begin{tabular}{|c|c|c|c|c|c|c|c|c|c|c|}
\hline \multirow[b]{3}{*}{ Gene name } & & & \multicolumn{6}{|c|}{ HFD-ASP (fold of Cont.) } & \multirow{2}{*}{\multicolumn{2}{|c|}{$\begin{array}{c}\begin{array}{c}\text { HFD-ELE } \\
\text { (fold of Cont.) }\end{array} \\
5 \%(n 6)\end{array}$}} \\
\hline & \multicolumn{2}{|c|}{ HFD-Cont. $(n 6)$} & \multicolumn{2}{|c|}{$0.03 \%(n 6)$} & \multicolumn{2}{|c|}{$0.1 \%(n 6)$} & \multicolumn{2}{|c|}{$0.3 \%(n 6)$} & & \\
\hline & Mean & SE & Mean & SE & Mean & SE & Mean & SE & Mean & SE \\
\hline \multicolumn{11}{|l|}{ GLUT } \\
\hline Glut4* ${ }^{*}+$ & 1.00 & 0.19 & 1.29 & 0.06 & 1.91 & 0.21 & $2 \cdot 20$ & 0.21 & $2 \cdot 29$ & 0.20 \\
\hline \multicolumn{11}{|l|}{ Glycolytic system } \\
\hline Hk2 & 1.00 & 0.07 & 0.91 & 0.08 & 1.01 & 0.14 & 0.95 & 0.09 & 0.83 & 0.08 \\
\hline \multicolumn{11}{|l|}{ TCA cycle } \\
\hline$C s^{*}+t$ & 1.00 & 0.05 & $1 \cdot 16$ & 0.07 & 1.32 & 0.12 & 2.09 & 0.08 & 1.90 & 0.05 \\
\hline $\operatorname{Idh} 3 \alpha^{*} \dagger$ & 1.00 & 0.09 & 1.14 & 0.07 & 1.29 & 0.08 & 1.71 & 0.09 & 1.57 & 0.10 \\
\hline Ogdh*t & 1.00 & 0.10 & 1.01 & 0.07 & $1 \cdot 71$ & 0.09 & $2 \cdot 23$ & 0.12 & $2 \cdot 42$ & 0.16 \\
\hline Sdha $(\text { Comp II })^{*} \dagger \neq$ & 1.00 & 0.12 & 1.42 & 0.07 & $2 \cdot 13$ & 0.19 & 2.54 & 0.13 & $2 \cdot 64$ & 0.16 \\
\hline \multicolumn{11}{|c|}{ Electron transfer system } \\
\hline Comp I*t‡ & 1.00 & 0.17 & 1.55 & 0.29 & $2 \cdot 11$ & 0.16 & $2 \cdot 18$ & 0.18 & 2.56 & 0.20 \\
\hline Comp IV*t‡ & 1.00 & $0 \cdot 10$ & 1.04 & 0.02 & 1.44 & 0.10 & 1.58 & 0.05 & 1.52 & 0.02 \\
\hline Comp IV* †t & 1.00 & 0.09 & $1 \cdot 10$ & 0.02 & 1.23 & 0.03 & 1.89 & 0.11 & 1.07 & 0.04 \\
\hline \multicolumn{11}{|l|}{ Fatty acid synthesis } \\
\hline Fas & 1.00 & 0.16 & 0.69 & 0.07 & 0.81 & 0.08 & 0.79 & 0.15 & 0.73 & 0.09 \\
\hline \multicolumn{11}{|l|}{ Fatty acid transporter } \\
\hline Fas & 1.00 & 0.14 & 1.37 & 0.17 & 1.15 & 0.12 & 1.01 & 0.12 & 1.04 & 0.08 \\
\hline \multicolumn{11}{|c|}{ Ketone bodies utilisation } \\
\hline $\mathrm{Scos}^{*} \dagger$ & 1.00 & 0.08 & 1.23 & 0.04 & $2 \cdot 13$ & 0.09 & $2 \cdot 27$ & 0.09 & 1.97 & 0.14 \\
\hline P3kcot ${ }^{*} \dagger$ & 1.00 & $0 \cdot 12$ & 1.09 & $0 \cdot 12$ & $2 \cdot 50$ & 0.12 & $2 \cdot 61$ & 0.15 & $2 \cdot 27$ & 0.17 \\
\hline \multicolumn{11}{|c|}{$\begin{array}{l}\text { Ketone body's utilisation } \\
\qquad \text { (acetoacetic acid } \rightarrow \text { acetyl CoA) }\end{array}$} \\
\hline Ogdh*枺 & 1.00 & 0.10 & 1.01 & 0.07 & $1 \cdot 71$ & 0.09 & $2 \cdot 23$ & 0.12 & 2.42 & 0.16 \\
\hline Sdha (Comp II)* $\dagger \ddagger$ & 1.00 & 0.12 & 1.42 & 0.07 & $2 \cdot 13$ & 0.19 & 2.54 & 0.13 & $2 \cdot 64$ & 0.16 \\
\hline
\end{tabular}

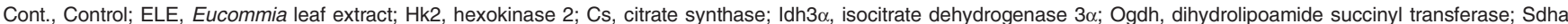
(Comp II), succinate dehydrogenase complex, subunit A, flavoprotein; Comp I, NADH dehydrogenase flavoprotein 1; Comp IV, cytochrome c oxidase, subunit 4a; Comp V, ATP synthase, $\mathrm{H}^{+}$transporting, mitochondrial $\mathrm{F} 1$ complex, delta subunit; Fas, fatty acid synthase; Fatp, fatty acid transporter protein; Scos, succinyl CoA synthase; P3kcot, peroxisomal 3-ketoacyl-CoA thiolase.

* Linear contrast with Control $(0 \%), 0.03 \%, 0.1 \%$ and $0.3 \%$ ASP $(P<0.05)$

† Contrast for Control v. $5 \%$ ELE $(P<0.05)$.

$\ddagger$ Contrast for the average of all ASP treatments v. $5 \%$ ELE $(P<0.05)$.

condition did not affect the hormone-sensitive lipase mRNA level in perirenal WAT (Table 3). Although the HFD-ASP groups had a clearly increased hormone-sensitive lipase mRNA level with linearity when compared with the HFD-Control group $(P<0.05)$, no difference was observed between the HFD-5\% ELE and the average of the HFD-0.03-0.3\% ASP groups (Table 3). The HFD-5\% ELE group exhibited marked suppression of mRNA expression of Fas in perirenal WAT $(P<0.05$, Table 3$)$. The HFD-ASP groups exhibited significantly decreased levels of Fas mRNA with linearity when compared with the HFD-Control group $(P<0.05)$ and these significant downregulations in the average of the HFD-0.03-0.3\% ASP groups were clearly lower than that of the HFD-5\% ELE group $(P<0 \cdot 05$, Table 3$)$. The $5 \%$ ELE group had significantly increased perirenal WAT Acadvl mRNA levels under HFD conditions $(P<0 \cdot 05$, Table 3). Although the HFD-ASP groups exhibited significantly increased levels of Acadvl mRNA with linearity when compared with the HFD-Control group $(P<0.05)$, no difference was also observed between the HFD-5\% ELE and the average of the three HFD-ASP groups $(P<0 \cdot 05$, Table 3$)$. There were no significant differences among any of the groups in the level of mRNA expression of Fas or FA transport protein in the Sol. M. of rats fed a HFD (Table 4).

\section{Gene expression related to ketone body metabolism with chronic administration of Eucommia leaf extract and asperuloside}

Chronic administration of $5 \%$ ELE led to significantly increased mRNA levels of succinyl CoA synthase, peroxisomal 3-ketoacyl-CoA thiolase, Ogdh and succinate dehydrogenase complex, subunit A, flavoprotein in the Sol. M. of rats under a HFD (each $P<0 \cdot 05$, Table 4). The HFD-ASP groups exhibited a significant up-regulation of these genes with linearity when compared with the HFD-Control group (each $P<$ $0 \cdot 05$, Table 4). No difference was not observed between the HFD-5 \% ELE and the average of all the HFD-0.03-0.3\% ASP groups (Table 4).

\section{PPAR $\gamma$, adiponectin and resistin gene expression with chronic} administration of Eucommia leaf extract and asperuloside

In the perirenal WAT of rats fed a HFD, chronic administration of $5 \%$ ELE led to a significant increase in PPAR $\gamma$ 
and adiponectin mRNA levels (each $P<0 \cdot 05$, Table 3). The ASP groups exhibited dose-dependent increases in the expression of both genes when compared with the HFD-Control rats $(P<0.05$, Table 3$)$. No difference in the increased PPAR $\gamma$ and adiponectin mRNA levels was observed between the HFD-5 \% ELE and the average of all HFD-ASP groups (Table 3). While 5\% ELE yielded significantly decreased resistin mRNA levels $(P<0.05$, Table 3$)$, no difference in the mRNA level was observed between the HFD-5\% ELE and the HFD-ASP groups (Table 3). The HFD-ASP groups exhibited significant down-regulation of the gene in a dose-dependent fashion when compared with the HFD-Control group $(P<0 \cdot 05$, Table 3$)$.

\section{Uncoupling protein gene expression with chronic administration of Eucommia leaf extract and asperuloside}

In the BAT of HFD-fed rats, chronic administration of $5 \%$ ELE led to significantly increased uncoupling protein 2 (UCP2) mRNA levels $(P<0 \cdot 05)$, but the $5 \%$ ELE administration did not affect the expression of other UCP mRNA (Table 5). Chronic 0.03-0.3\% ASP administration clearly increased the mRNA levels of UCP1 and UCP2 in a dosedependent fashion when compared with the HFD-Control group (each $P<0 \cdot 05$, Table 5). No difference in the increased UCP1 and UCP2 mRNA levels was observed between the HFD-5\% ELE and the average of the three HFD-ASP groups (Table 5).

\section{Hepatic lipid metabolic enzyme activity with chronic administration of Eucommia leaf extract and asperuloside}

In rats under HFD conditions, chronic administration of $5 \%$ ELE led to a significant increase in mitochondrial Cpt1 $\alpha$ activity in the liver compared with the HFD-Control group $(P<0 \cdot 05$, Fig. 1(a)). Moreover, chronic administration of ASP dose-dependently increased the mitochondrial Cpt1 $\alpha$ activity in the liver of rats under HFD conditions when compared with the HFD-Control rats $(P<0 \cdot 05$, Fig. 1(a)). In contrast, significant changes in cytosolic Fas activity could not be observed during RT-PCR analysis in ELE and ASP groups (Fig. 1(b)).
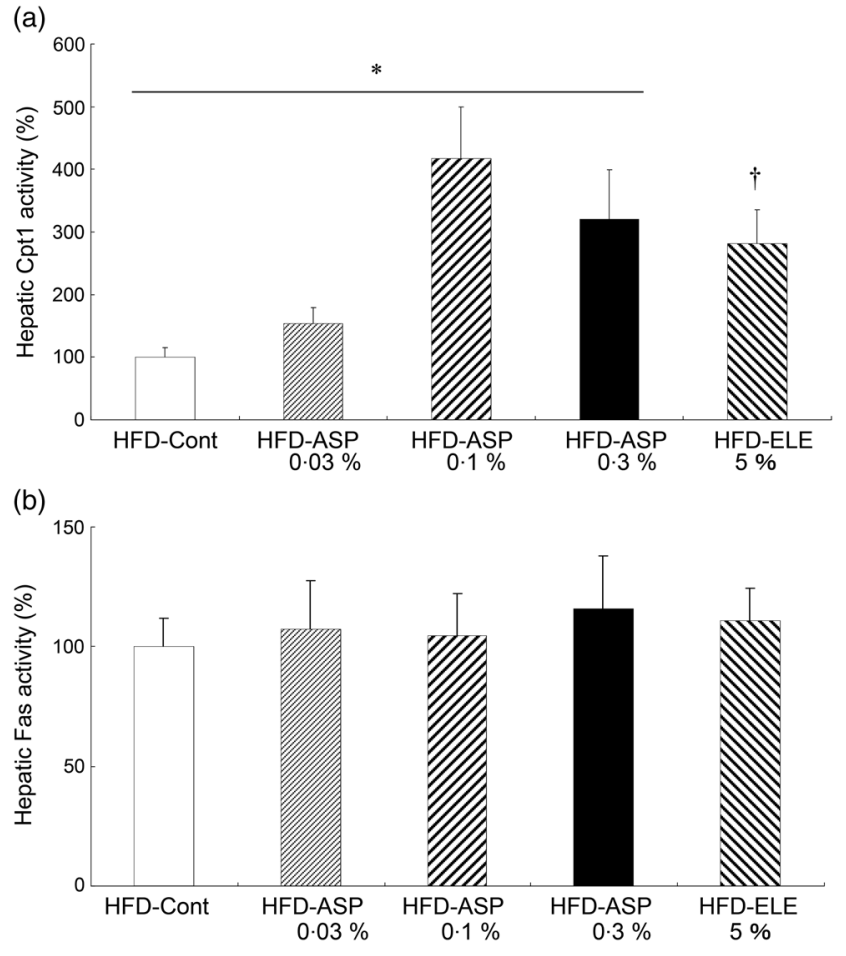

Fig. 1. Effect of asperuloside (ASP) on the activity of hepatic carnitine palmitoyltransferase $1 \alpha(\mathrm{Cpt} 1 \alpha)$ (a) and hepatic fatty acid synthase (fas) (b) in high-fat diet (HFD)-fed rats. Values are means $(n 6)$ with standard errors represented by vertical bars. ELE, Eucommia leaf extract. * Quadratic contrast with Control $(0 \%), 0.03,0.1$ and $0.3 \%$ ASP $(P<0.05)$. † Contrast for Control v. $5 \% \operatorname{ELE}(P<0.05)$.

\section{Discussion}

Similarly to our previous study ${ }^{(3)}, 3$-month administration of ASP controlled the body weight gain, food intake and relative WAT weight and increased the relative BAT weight with prominently decreased plasma levels of TAG, NEFA and total cholesterol in HFD rats. The administration of ASP at a dose of $0.1 \%$ exhibited effects comparable to those observed with the administration of ELE at a dose of $5 \%$. The effects were maintained at a dose of $0.3 \%$. Moreover, the results agreed with previous findings that showed that ASP but not GEA and CHA decreases the body weight and WAT weight

Table 5. Gene expression analysis by real-time PCR in brown adipose tissue after administration of asperuloside (ASP) under high-fat diet (HFD) conditions (Mean values with their standard errors)

\begin{tabular}{|c|c|c|c|c|c|c|c|c|c|c|}
\hline \multirow[b]{3}{*}{ Gene name } & \multirow{2}{*}{\multicolumn{2}{|c|}{ HFD-Cont. $(n 6)$}} & \multicolumn{6}{|c|}{ HFD-ASP (fold of Cont.) } & \multirow{2}{*}{\multicolumn{2}{|c|}{$\begin{array}{c}\begin{array}{c}\text { HFD-ELE } \\
\text { (fold of Cont.) }\end{array} \\
5 \%(n 6)\end{array}$}} \\
\hline & & & \multicolumn{2}{|c|}{$0.03 \%(n 6)$} & \multicolumn{2}{|c|}{$0.1 \%(n 6)$} & \multicolumn{2}{|c|}{$0.3 \%(n 6)$} & & \\
\hline & Mean & $\mathrm{SE}$ & Mean & $\mathrm{SE}$ & Mean & SE & Mean & $\mathrm{SE}$ & Mean & $\mathrm{SE}$ \\
\hline \multicolumn{11}{|c|}{$\begin{array}{l}\text { Uncoupling ATP synthesis from } \\
\text { oxidative metabolism }\end{array}$} \\
\hline UCP1 ${ }^{*}$ & 1.00 & 0.07 & 0.98 & 0.04 & 1.38 & 0.10 & 1.98 & 0.07 & 1.01 & 0.05 \\
\hline UCP2* $†$ & 1.00 & 0.08 & $1 \cdot 10$ & 0.07 & 1.42 & 0.13 & 1.64 & 0.07 & $2 \cdot 26$ & 0.14 \\
\hline UCP3 & 1.00 & 0.07 & 0.96 & 0.06 & 0.92 & 0.06 & 0.89 & 0.04 & 1.09 & 0.02 \\
\hline
\end{tabular}

Cont., Control; ELE, Eucommia leaf extract; UCP, uncoupling protein.

${ }^{*}$ Linear contrast with Control $(0 \%), 0.03 \%, 0.1 \%$ and $0.3 \%$ ASP $(P<0.05)$.

† Contrast for Control v. $5 \%$ ELE $(P<0.05)$. 
via significantly reduced circulating NEFA levels in HFD-fed mice $^{(20)}$.

For carbohydrate metabolism, no difference in plasma glucose level was observed in rats administered ELE under HFD conditions ${ }^{(3)}$. On the other hand, some studies reported that ELE controls the plasma glucose level in type 1 diabetes model rats and type 2 diabetes model mice (C57BL/KsJ-db/ $\mathrm{db}$ mice $)^{(23,24)}$. The HFD model animals, which were employed in this study, showed a characteristic increase in plasma insulin levels, and the magnitude of this increase was considerably decreased by ELE administration over 3 months ${ }^{(3)}$. Our study showed that $5 \%$ ELE administration decreased the plasma level of insulin under HFD conditions, and a significant and dose-dependent decrease was observed in rats fed $0.03-0.3 \%$ ASP, resulting in a significant decrease in plasma glucose level in a dose-dependent fashion. This suggests that ASP may improve insulin resistance in rat models of HFD-induced obesity. RT-PCR analysis showed that chronic administration of ASP to rats under a HFD significantly increased the mRNA levels of Cs, Idh3 $\alpha$, Ogdh, Sdha, Comp I, Comp IV and Comp V in skeletal muscles. Therefore, there is a possible increase in the abundance of enzymes in these pathways that could increase the capacity of the glycolytic system, tricarboxylic acid cycle and electron transport system in skeletal muscles, increasing the mRNA levels of glucokinase, Cs, Ogdh, Comp I and Comp IV in the liver and markedly decreasing the mRNA levels of Idh3 $\alpha$ and Comp I in the WAT. GLUT4 mRNA expression was increased in skeletal muscle and decreased in WAT under the same dietary conditions. In the HFD model rats, the effect of ASP on carbohydrate metabolism resembled that of ELE, supporting the results of a previous study ${ }^{(3)}$. Thus, ASP may be an important component of ELE responsible for the control of carbohydrate metabolism. Similar to ELE-fed rats, the ASP-fed animals might no longer be able to maintain the shape of the adipose cells that store TAG because of the dose-dependently decreased ATP production in the WAT under HFD conditions. A diminished perirenal WAT cell size was observed in rats fed ELE and ASP under HFD conditions (preliminary histological results of perirenal WAT cells showed that the range of most adipose cell areas $\left(\mu \mathrm{m}^{2}\right)$ and the occupancy (\%) in the cells within view $(n 4)$ are as follows: HFD-Control, 2001-42 $000 \mu^{2} / 94.6 \%$; HFD-5 \% ELE， 2001-24 $000 \mathrm{\mu m}^{2} / 92.2 \%$; HFD-0.3\% ASP, $1-8000 \mu \mathrm{m}^{2} / 91.5 \%$ ). The ASP (ELE, $P<0.05$ ) groups might increase the use of glucose in skeletal muscles, decreasing the circulating glucose level under HFD conditions.

One study showed that increases in plasma levels of TAG, NEFA and total cholesterol are directly related to higher incidences of obesity and other lipid-associated diseases ${ }^{(25)}$. In a recent study, 3-month administration of 9\% ELE suppressed plasma TAG levels in rats under HFD conditions ${ }^{(3)}$. A hypolipidaemic effect of ELE has been reported ${ }^{(17)}$ in which ELE significantly attenuates the increased TAG level in plasma following a single administration of lipids. Eucommia leaves stimulated liver lipid metabolism and decreased the plasma level of TAG and total cholesterol in hamsters ${ }^{(23)}$. In addition, a recent study showed that ELE might affect the control of absorption of TAG derived from foods into the bloodstream as well as the activation of lipid metabolism in the liver, in particular fatty acid (FA) $\beta$-oxidation ${ }^{(3)}$. In this study, chronic administration of $5 \%$ ELE led to pronounced decreases in plasma levels of NEFA, TAG and total cholesterol under HFD conditions, and significant and dose-dependent decreases were observed in rats administered ASP, suggesting that chronic administration of ELE and ASP may improve hyperlipidaemia in rats with HFD-induced obesity. Furthermore, RT-PCR analysis showed that chronic administration of ASP dosedependently increased the level of FA transport protein, Cpt1 $\alpha$ and Acadvl mRNA and suppressed the level of Fas mRNA in the liver of rats on a HFD, as well as ELE groups. The results show that there is a possible increase in the abundance of enzymes in pathways that could increase the capacity for FA $\beta$-oxidation. In HFD-fed rats, uptake of FA into the liver was increased by the administration of either ASP or ELE. The uptake was followed by an increased FA $\beta$-oxidation and ATP production, which decreased plasma NEFA levels. In contrast, the lipid metabolic enzyme activity assay revealed that the administration of ASP or ELE stimulated mitochondrial Cpt1 $\alpha$ activity in the liver but not Fas activity in the cytosol. Although we cannot conclude that ASP and ELE directly promote FA $\beta$-oxidation, the FA transport system, which reaches the mitochondrion before $\beta$-oxidation, is activated. These results, in addition to the results of the RT-PCR analysis and the determination of lipid plasma levels, show that ASP and ELE have lipid metabolic-stimulating effects that may be influenced by the activation of FA $\beta$-oxidation. Concomitantly, significant activation of lipolysis was observed in the WAT of rats supplemented with ASP; however, a similar effect was not observed in animals fed HFD-5\% ELE. Similar to chronic administration of ELE (in this study and a previous study $)^{(3)}$, chronic administration of ASP significantly induced mRNA expression of PPAR $\gamma$ and adiponectin, which may depend on the accumulation of visceral fat to improve insulin resistance or hyperlipaemia. The increased expression of these mRNA may activate FA $\beta$-oxidation in the WAT of rats under HFD conditions. In this study, chronic administration of ASP and ELE was found to significantly decrease the plasma levels of NEFA and TNF- $\alpha$ and significantly increase the plasma level of adiponectin. One study reported that ELE suppressed hepatic activities of Fas and 3-hydroxy-3-methylglutaryl (HMG)-CoA reductase and increased hepatic $\beta$-oxidation activity in HFD-fed hamsters ${ }^{(23)}$, resulting in decreased plasma levels of FA and cholesterol as well as hepatic levels of cholesterol. Moreover, our results showed that chronic administration of any tested dose of ASP or ELE caused simultaneous activation of ketone bodies use in skeletal muscle and hepatic FA $\beta$-oxidation in rats under HFD conditions.

$\mathrm{UCP}^{(26)}$, of which at least three closely related homologues have been cloned, are FA anion transporters in addition to proton transporters ${ }^{(27)}$. UCP1 (thermogenin) mediates nonshivering thermogenesis in the BAT, uncoupling the respiratory chain and allowing fast substrate oxidation with a low rate of ATP generation ${ }^{(28)}$. Interestingly, chronic administration of ASP at a dose from 0.03 to $0.3 \%$ increased the BAT UCP1 
mRNA level in a dose-dependent fashion under HFD conditions; in contrast, such an effect was not observed when administering $5 \%$ ELE. Thus, based on the RT-PCR analysis of gene expression, the up-regulation of UCP1 mRNA expression by ASP was attenuated by other ingredients in the ELE. Chronic administration of ASP and ELE increased the UCP2 mRNA level in the BAT of rats under HFD conditions. One study showed that the skeletal muscle UCP3 mRNA level was unchanged, but the UCP3 protein level was significantly increased in conjugated linoleic acid-fed mice ${ }^{(29)}$. Therefore, UCP protein expression may be translationally or posttranslationally regulated. Our recent results showed that administration of $0.1 \%$ ASP for 1 month significantly enhanced the BMR and decreased the respiratory quotient, which might stimulate lipid metabolisms in rats fed a $\operatorname{HFD}^{(20)}$.

In conclusion, chronic administration of ASP systemically stimulated metabolic function in rats across several organs. ASP administration resulted in decreased ATP production in the WAT, accelerated FA $\beta$-oxidation in the liver and increased use of ketone bodies and glucose in skeletal muscle and may have increased non-shivering thermogenesis due to UCP1 expression in the BAT. Thus, ASP exerted anti-obesity and anti-metabolic syndrome effects. This study showed that ASP may act as a major ingredient in ELE, relating nearly every metabolic function across several organs, except for the activation of the BAT UCP1-induced thermogenesis.

\section{Acknowledgements}

The authors are grateful to M. Mikame for technical assistance and statistical analysis, S. Nakao for animal care and Dr M. Yamada for special advice as a statistician. The study was performed with support from Kobayashi Pharmaceutical Co. Ltd. ELE and ASP were provided by Kobayashi Pharmaceutical Co. Ltd. The study sponsors had no role in the design and conduct of the study; the collection, management, analysis and interpretation of the data; or the preparation, approval and submission of the manuscript. All the authors contributed to the writing of the manuscript and the interpretation of the results. They reviewed the contents and approved the final version submitted for publication. T. F. was the principal investigator of the study and supervised the data collection; T. H., A. W., N. K., T. U., Y. Y., H. S., T. M., A. Y., M. O. and S. N. contributed to the design of the study, data collection and laboratory analysis; and Y. Y. contributed to laboratory analysis. There are no financial or personal conflicts of interest in relation to the present study.

\section{Supplementary material}

The supplementary material for this article can be found at http://journals.cambridge.org/JNS

\section{References}

1. Matsuzawa Y (2005) Adiponectin identification, physiology and clinical relevance in metabolic and vascular disease. Atheroscler Suppl 6, 7-14.
2. Bray GA (2008) Lifestyle and pharmacological approaches to weight loss: efficacy and safety. J Clin Endocrinol Metab 93, S81S88. [Review. Erratum in: J Clin Endocrinol Metab (2009) 94, 324.]

3. Fujikawa T, Hirata T, Wada A et al. (2010) Chronic administration of Eucommia leaf stimulates metabolic function of rats across several organs. Br J Nutr 104, 1868-1877.

4. Jiangsu New Medical College (editor) (1997) Chinese Materia Medica Dictionary, p. 1031. Shanghai: Shanghai Science and Technology Publishing House.

5. Nakamura T, Nakazawa Y, Onizuka S, et al. (1997) Studies on the constituents of Eucommia ulmoides iridoids from the leaves. Nat Med 51, 275-277.

6. Li H, Chen B \& Yao S (2005) Application of ultrasonic technique for extracting chlorogenic acid from Eucommia ulmodies Oliv. Ultrason Sonochem 12, 295-300.

7. Qin ZD, Wu YZ, Yu ZQ, et al. (1997) Studies on the Eucommia cortex and leaves. West-North Univ J 7, 64-71.

8. Guizhou Province Institute for Drug Control and Guizhou Province Chinese Medicine Research Laboratories (1978) A hypertensive clinical study on Eucommia leaves. Chin Med Herbs Res 8, 59-63.

9. Guizhou Province Hypotensive Clinical Committee Institute for Drug Control and Chinese Medicine Research Laboratories (1978) A clinical study on replacement of Eucommia cortex with Eucommia leaves. New Med J 10, 30-32.

10. Liu D \& Li CW, (1980) Clinical evaluation of Eucommia cortex and leaves by 102 type of hypertension. Shanxi Chin Med 1, 27-30.

11. Shanxi Province Eucommia Clinical Review Committee (1995) Replacement of Eucommia Cortex with Eucommia Leaves for Hypertension Treatment. A Clinical Review with 621 Cases, pp. 19-54. Xian: Shanxi Eucommia Development Company.

12. Yamaguchi Y, Kawamura N, Tsuboi T, et al. (2007) Effect of Eucommia ulmoides leaf extract on blood pressure. Int Symp Eucommia ulmoides 1, 55-62.

13. Tagawa C, Nakazawa Y, Tagashira E, et al. (2005) Effect of Eucommia leaf (Eucommia ulmoides Oliver; Du-Zhong yge) extract on blood pressure(2). Nat Med 59, 117-120.

14. Ohmori R, Iwamoto T, Tago M, et al. (2005) Antioxidant activity of various teas against free radicals and LDL oxidation. Lipids 40 , 849-853.

15. Yang J, Kato K, Noguchi K, et al. (2003) Tochu (Eucommia ulmoides) leaf extract prevents ammonia and vitamin $\mathrm{C}$ deficiency induced gastric mucosal injury. Life Sci 73, 3245-3256.

16. Fujikawa T, Tsuboi T, Kawamura N, et al. (2007) Eucommia ulmoides Oliver as a prophylactic for restraint stress in water-induced gastric erosion in rats. Int Symp Eucommia ulmoides 1, 67-71.

17. Ando C, Kobayashi T, Tsukamoto S, et al. (2007) Antiobesity effects of Eucommia ulmoides leaves. Int Symp Eucommia ulmoides 1, 63-66.

18. Metori K, Ohashi S, Takahashi S, et al. (1994) Effects of Du-Zhong leaf extract on serum and hepatic lipids in rats fed a high-fat diet. Biol Pharm Bull 17, 917-920.

19. Choi MS, Jung UJ, Kim HJ, et al. (2008) Du-Zhong (Eucommia ulmoides Oliver) leaf extract mediates hypolipidemic action in hamsters fed a high-fat diet. Am J Chin Med 36, 81-93.

20. Hirata T, Kobayashi T, Wada A, et al. (2011) Anti-obesity compounds in green leaves of Eucommia ulmoides. Bioorg Med Chem Lett 21, 1786-1791.

21. Kelley DS, Nelson GJ \& Hunt JE, (1986) Effect of prior nutritional status on the activity of lipogenic enzymes in primary monolayer cultures of rat hepatocytes. Biochem J 235, 87-90.

22. Markwell MA, McGroarty EJ, Bieber LL, et al. (1973) The subcellular distribution of carnitine acyltransferases in mammalian liver and kidney. A new peroxisomal enzyme. J Biol Chem 248, 34263432.

23. Lee MK, Kim MJ, Cho SY, et al. (2005) Hypoglycemic effect of Du-Zhong (Eucommia ulmoides Oliv.) leaves in streptozotocininduced diabetic rats. Diabetes Res Clin Pract 67, 22-28. 
24. Park SA, Choi MS, Kim MJ, et al. (2006) Eucommia ulmoides Oliver leaf extract increases endogenous antioxidant activity in type 2 diabetic mice. J Med Food 9, 474-479.

25. Palou A, Pico C, Bonet M, et al. (1998) The uncoupling protein, thermogenin. Int J Biochem Cell Biol 30, 7-11.

26. Samec S, Seydoux J \& Dulloo AG, (1998) Role of UCP homologues in skeletal muscles and brown adipose tissue: mediator of thermogenesis or regulators of lipids as fuel substrate? FASEB J $12,715-724$.

27. Ricquier D, Thibault J, Bouillaud F, et al. (1983) Molecular approach to thermogenesis in brown adipose tissue. Cell-free translation of mRNA and characterization of the mitochondrial uncoupling protein. J Biol Chem 258, 6675-6677.

28. Petrovic N, Walden TB, Shabalina IG, et al. (2010) Chronic peroxisome proliferator-activated receptor gamma (PPARgamma) activation of epididymally derived white adipocyte cultures reveals a population of thermogenically competent, UCP1-containing adipocytes molecularly distinct from classic brown adipocytes. J Biol Chem 285, 7153-7164.

29. Ealey KN, EI-Sohemy A \& Archer MC (2002) Effects of dietary conjugated linoleic acid on the expression of uncoupling proteins in mice and rats. Lipids 37, 853-861. 